\title{
Zur Entwicklung des ostdeutschen Arbeitsmarktes im Aufschwung
}

Hans-Ulrich Brautzsch

Vom jüngsten konjunkturellen Aufschwung in Deutschland hat der ostdeutsche Arbeitsmarkt sichtbar profitiert. Die Zahl der Erwerbstätigen hat kräftig zugenommen und die Unterbeschäftigung ging deutlich zurück. Damit zeigt sich eine gänzlich andere Entwicklung als beim vorangegangenen Aufschwung, bei dem sich die Lage auf dem ostdeutschen Arbeitsmarkt sogar noch verschlechtert hatte. Gleichwohl ist die Arbeitsplatzlücke in Ostdeutschland immer noch sehr viel höher als in Westdeutschland Der Beitrag zeichnet nach, in welchem Umfang sich die Situation des ostdeutschen Arbeitsmarktes im Vergleich zu Westdeutschland verbessert hat, wie viele Arbeitsplätze zur Verfügung stehen und wie viele noch immer fehlen.

\section{Untersuchungsgegenstand und Methode}

Der kräftige konjunkturelle Aufschwung der Jahre 2005 bis 2007 hat auch den ostdeutschen Arbeitsmarkt erreicht (Arbeitskreis Konjunktur Ostdeutschland 2008; Projektgruppe Gemeinschaftsdiagnose 2008). So nahm in Ostdeutschland ${ }^{1}$ die Zahl der Erwerbstätigen das zweite Jahr in Folge zu und die Unterbeschäftigung ging deutlich zurück. Einige Aspekte dieser Entwicklung am ostdeutschen Arbeitsmarkt im gegenwärtigen konjunkturellen Aufschwung vertiefend zu analysieren, ist Gegenstand dieses Beitrages. Als Vergleichsmaßstab wird zum einen der vorangegangene Aufschwung (1998-2000) herangezogen. Zum anderen dient die Entwicklung in Westdeutschland als Referenzlinie für die Beurteilung der ostdeutschen Entwicklung.

Der Beitrag ist wie folgt aufgebaut: In einem ersten Schritt wird anhand der Indikatoren Erwerbstätigenzahl, Arbeitsvolumen und Anzahl der Unterbeschäftigten analysiert, in welchem Umfang sich die Lage auf dem ostdeutschen Arbeitsmarkt im gegenwärtigen Aufschwung verbessert hat. In einem zweiten Schritt wird untersucht, wie sich der Beschäftigungsstand bei den einzelnen Beschäftigtengruppen, wie beispielsweise bei den sozialversicherungspflichtig Beschäftigten und bei den Selbstständigen, entwickelt hat. Danach wird aufgezeigt, wie sich im gegenwärtigen konjunkturellen Aufschwung die Arbeitsplatzausstattung, also die Zahl der Arbeitsplätze bezogen auf 1.000 Erwerbsfähige, verbessert hat. ${ }^{2}$ Abschließend wird gezeigt, dass trotz der Verbesserung der Arbeitsplatzaus- stattung die Arbeitsplatzlücke, d.h. die Zahl der fehlenden Arbeitsplätze, nach wie vor (zu) hoch ist.

Beim Zyklenvergleich werden üblicherweise Quartalsangaben für die relevanten Konjunkturindikatoren verwendet (Horn et al. 2008; SVR 2007; Ludwig 2008). Die vorliegende Analyse basiert hingegen analog der Untersuchung des Instituts für Arbeitsmarkt- und Berufsforschung (IAB) (Bach et al. 2007) - auf Jahresangaben. Dies ist insbesondere darauf zurückzuführen, dass für die beiden Großraumregionen keine für die Analyse geeigneten amtlichen Quartalsdaten, zum Beispiel für das Bruttoinlandsprodukt, die Arbeitnehmer, die Selbstständigen und das Arbeitsvolumen, vorliegen. Da eine abschließende Bewertung des jüngsten Aufschwungs derzeit noch nicht erfolgen kann, ja seine weitere Entwicklung aktuell höchst ungewiss ist, sind die Wertungen und Schlussfolgerungen vorläufiger Art.

\section{Merkliche Besserung auf dem ostdeutschen Arbeitsmarkt}

Die wichtigsten Indikatoren zur Beurteilung der Arbeitsmarktsituation weisen für West- wie für Ostdeutschland auf eine Besserung hin (Tabelle 1 und 2). Für den ostdeutschen Arbeitsmarkt gilt (Tabelle 1): Die Zahl der Erwerbstätigen (Inland) stieg im Zeitraum zwischen 2005 und 2007 um 2,1 \%; die Zahl der Erwerbstätigen (Inländer) nahm mit der gleichen Rate zu. ${ }^{3}$ Das Arbeitsvolumen - dies ist die tatsächlich geleistete Arbeitszeit aller Erwerbstätigen in einem Jahr - stieg um 1,7 \% an. Damit steigerten sich im gegenwärtigen Aufschwung die Zahl der Erwerbstätigen und das Arbeitsvolumen in Ostdeutschland etwas verhaltener als in Westdeutschland. Die Unterbeschäftigung, zu der die registrierten Arbeitslosen und die sonstigen Unterbeschäftigten in arbeitsmarktpolitischen Maßnahmen gehören, ging im Osten mit etwa der gleichen Rate wie in Westdeutschland zurück.

Der vorangegangene konjunkturelle Aufschwung hingegen ging am ostdeutschen Arbeitsmarkt vorbei: Trotz des kräftigen Zuwachses der gesamtwirtschaftlichen Produktion nahmen seinerzeit die Zahl der Erwerbstätigen und das Arbeits-

\footnotetext{
1 Unter Ostdeutschland werden hier die Länder Brandenburg, Mecklenburg-Vorpommern, Sachsen, Sachsen-Anhalt und Thüringen subsumiert. Westdeutschland umfasst das frühere Bundesgebiet einschließlich Berlin.

2 Unter der Arbeitsplatzausstattung wird im Folgenden die Zahl der Erwerbstätigen je 1000 Erwerbsfähige verstanden. Erwerbsfähige sind Personen im Alter von 15 bis 64 Jahren.

3 Die Zahl der Erwerbstätigen (Inland) stellt die Erwerbstätigen entsprechend ihres Arbeitsortes dar. Die Zahl der Erwerbstätigen (Inländer) erfasst sie an ihrem Wohnort. So gab es im Jahr 2007 in Ostdeutschland ca. 5,7 Mio. Erwerbstätige (Inland) Die Zahl der Erwerbstätigen (Inländer) betrug hingegen etwa 6,1 Mio. Die Differenz zwischen beiden Größen ist der sogenannte Pendlersaldo, der die Differenz zwischen der Zahl der Auspendler und der Einpendler angibt.
}

Hans-Ulrich Brautzsch, Dr., Wissenschaftler im Institut für Wirtschaftsforschung Halle (IHW), Abteilung Makroökonomik. Arbeitsschwerpunkte: Konjunkturforschung, Arbeitsmarktökonomie, Input-OutputAnalyse.

e-mail: Ulrich.Brautzsch@iwh-halle.de 
volumen sogar noch ab und die Unterbeschäftigung stieg. Die Zunahme der Unterbeschäftigung wäre noch höher ausgefallen, wenn nicht in diesem Zeitraum die Zahl der Ostdeutschen, die nach Westdeutschland pendeln, deutlich zugenommen hätte. Dies zeigt sich daran, dass die Zahl der Erwerbstätigen (Inländer) um $0,8 \%$ zugenommen hatte. Im Unterschied $\mathrm{zu}$ Ostdeutschland verbesserte sich hingegen die Situation auf dem westdeutschen Arbeitsmarkt auch damals erheblich. Dies weisen sowohl der kräftige Anstieg der Erwerbstätigkeit und des Arbeitsvolumens als auch der Rückgang der Unterbeschäftigung aus.

Doch blicken wir wieder auf den Aufschwung der Jahre 2005 bis 2007: Hier hat Ostdeutschland am Beschäftigungsaufbau etwas unterproportional partizipiert. Als Maßstab dient dabei der Anteil der ca. 8,9 Mio. ostdeutschen Erwerbsfähigen am gesamtdeutschen Wert. Er betrug im Jahr 2007 ca. $16 \%$. Mit ca. 13 \% war der ostdeutsche Anteil beim Zuwachs der Erwerbstätigkeit etwas niedriger (Tabelle 3). Beim Arbeitsvolumen sind es etwa $12 \%$. Hingegen war der Anteil Ostdeutschlands am Rückgang der Unterbeschäftigung deutlich höher. Die entscheidende Ursache hierfür ist der starke Rückgang der Zahl der Erwerbsfähigen. Dies ist zum einen durch die demografische Entwicklung bedingt: Infolge des Eintritts in das gesetzliche Rentenalter schieden in Ostdeutschland zwischen 2005 und 2007 ca. 210.000 Personen mehr aus dem Arbeitsmarkt aus, als junge Erwerbsfähige (also Jugendliche, die das 15. Lebensjahr erreicht haben) hinzukamen. Zum anderen überstiegen in diesem Zeitraum die Abwanderungen aus den östlichen Bundesländern nach Westdeutschland die Zuzüge aus den alten Bundesländern um ca. 150.000 Personen (Kubis/ Schneider 2008, S. 128ff.; IAB 2008).

Betrachtet man die Entwicklung der Erwerbstätigkeit nach Beschäftigtengruppen, so wird sichtbar, dass sowohl in Ostals auch in Westdeutschland der Beschäftigungsaufbau nur zu einem kleinen Teil auf staatlich geförderte Arbeitsplätze entfällt (Tabelle 4 und 5). Vielmehr nahm die Zahl der nicht geförderten sozialversicherungspflichtig Beschäftigten und der Selbstständigen kräftig zu. Bemerkenswert ist auch, dass im gegenwärtigen Aufschwung auch in Ostdeutschland mehr Vollzeitarbeitsplätze angeboten und nicht wie im vorangegangenen Aufschwung -

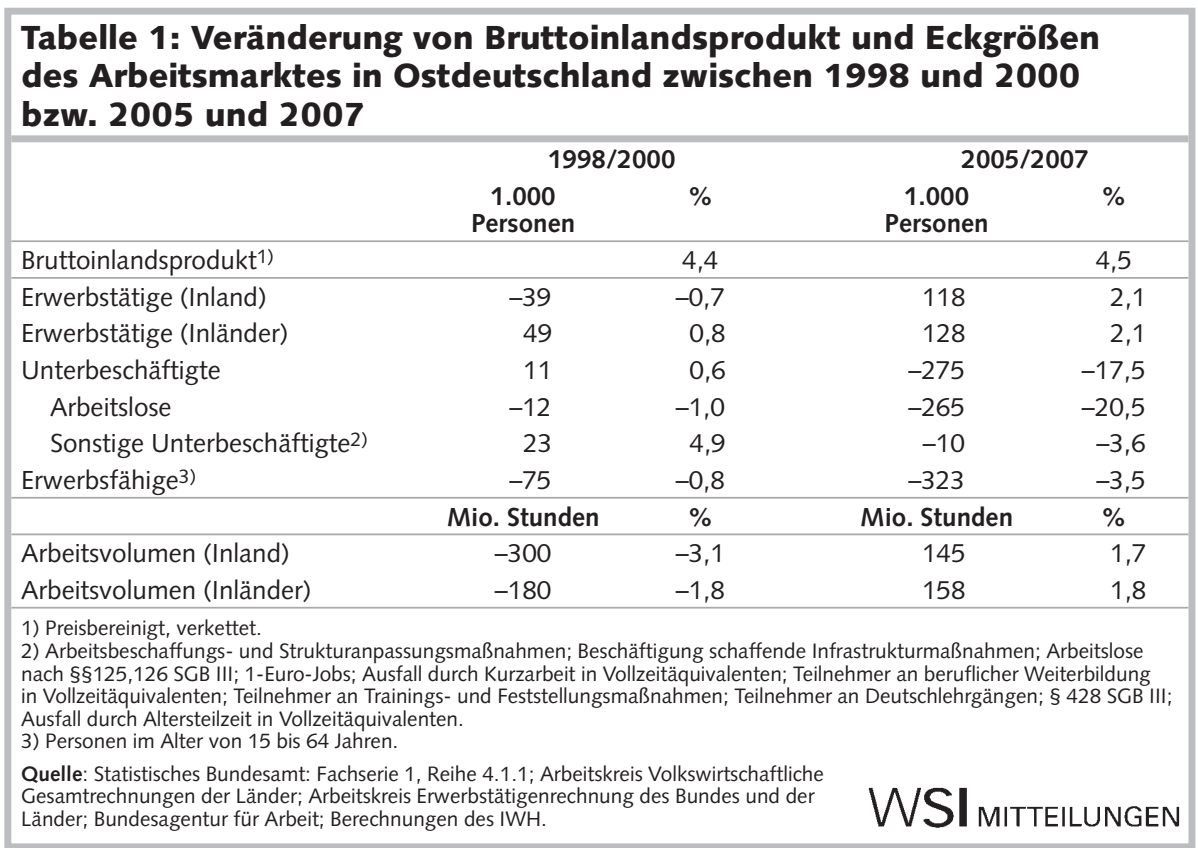

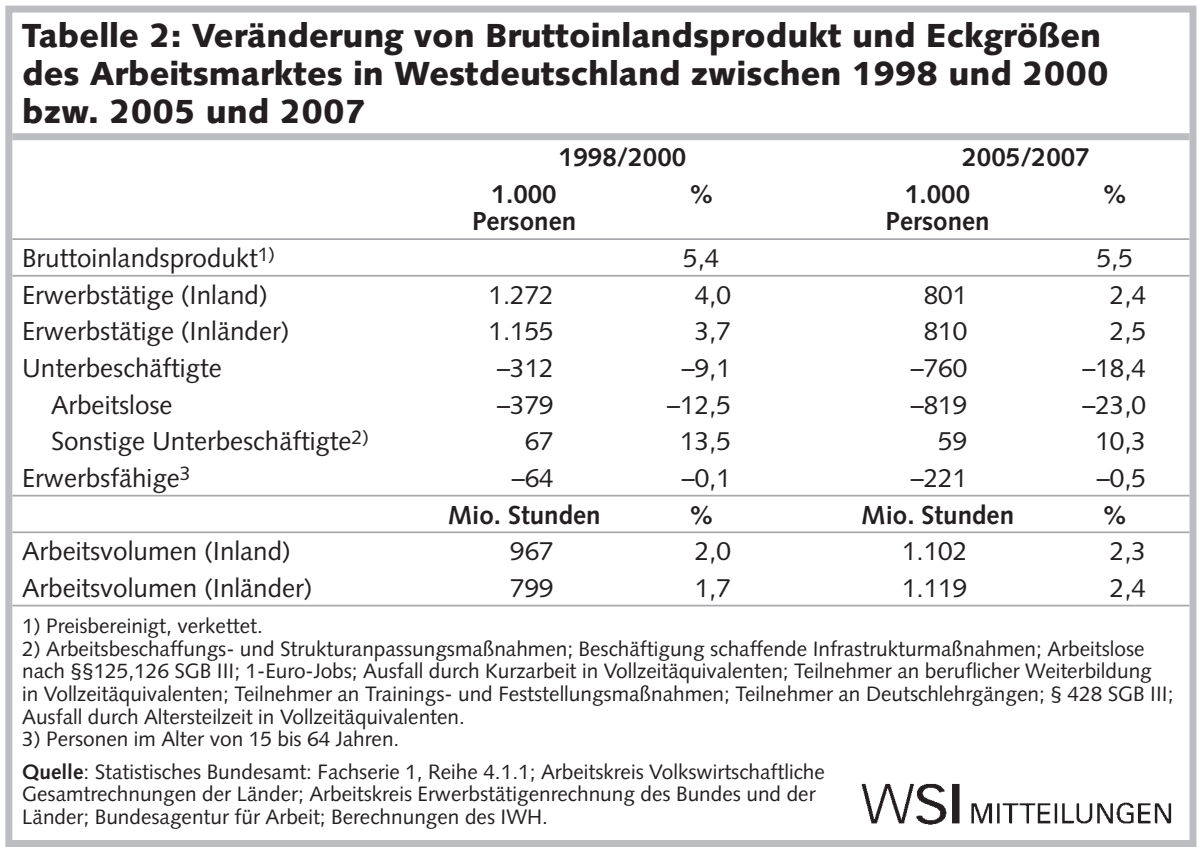

Tabelle 3: Anteil von Ost- und Westdeutschland an der Veränderung von Eckwerten des Arbeitsmarktes in Deutschland von 2005 bis 2007

\begin{tabular}{|c|c|c|c|}
\hline & \multirow{2}{*}{$\begin{array}{c}\text { Deutschland } \\
\text { insgesamt }\end{array}$} & \multicolumn{2}{|c|}{ Anteil von ... an Deutschland insgesamt } \\
\hline & & Ostdeutschland & Westdeutschland \\
\hline & 1.000 Personen & $\%$ & $\%$ \\
\hline Erwerbstätige (Inland) & 919 & 12,9 & 87,1 \\
\hline Erwerbstätige (Inländer) & 938 & 13,6 & 86,4 \\
\hline $\begin{array}{l}\text { Unterbeschäftigte } \\
\text { darunter: }\end{array}$ & -1.036 & 26,3 & 73,7 \\
\hline Arbeitslose & -1.085 & 24,4 & 75,6 \\
\hline Erwerbsfähige ${ }^{1}$ & -544 & 59,4 & 40,6 \\
\hline & Mio. Stunden & $\%$ & $\%$ \\
\hline Arbeitsvolumen (Inland) & 1.247 & 11,6 & 88,4 \\
\hline Arbeitsvolumen (Inländer) & 1.172 & 13,5 & 86,5 \\
\hline
\end{tabular}




\begin{tabular}{|c|c|c|c|c|}
\hline \multicolumn{5}{|c|}{$\begin{array}{l}\text { Tabelle 4: Veränderung der Zahl der Erwerbstä } \\
\text { Beschäftigtengruppen in Ostdeutschland zwis } \\
\text { bzw. } 2005 \text { und } 2007\end{array}$} \\
\hline 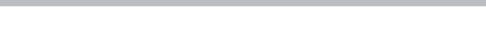 & \multicolumn{2}{|c|}{$1998 / 2000$} & \multicolumn{2}{|c|}{$2005 / 2007$} \\
\hline & $\begin{array}{l}1.000 \\
\text { Personen }\end{array}$ & $\%$ & $\begin{array}{l}1.000 \\
\text { Personen }\end{array}$ & $\%$ \\
\hline $\begin{array}{l}\text { Arbeitnehmer (Inland) } \\
\text { darunter: }\end{array}$ & -68 & $-1,2$ & 102 & 2,0 \\
\hline Sozialversicherungspflichtig Beschäftigte & -147 & $-3,1$ & 107 & 2,7 \\
\hline Gefördert $\left.{ }^{1}\right)$ & 18 & 8,8 & -10 & $-12,3$ \\
\hline Nicht gefördert & -165 & $-3,7$ & 117 & 3,0 \\
\hline Vollzeit & -161 & $-3,9$ & 58 & 1,7 \\
\hline Teilzeit & 14 & 2,3 & 49 & 7,9 \\
\hline Ausschließlich geringfügig Beschäftigte & 233 & 138,9 & 13 & 2,5 \\
\hline $\begin{array}{l}\text { 1-Euro-Job } \\
\text { (Variante: Mehraufwandsentschädigung) }\end{array}$ & & & 13 & 17,5 \\
\hline $\begin{array}{l}\text { Selbstständige einschließlich } \\
\text { mithelfender Familienangehöriger }\end{array}$ & 29 & 5,8 & 17 & 2,7 \\
\hline Geförderte Selbstständige2) & -1 & $-9,8$ & -18 & $-21,3$ \\
\hline Nicht geförderte Selbstständige & 30 & 6,3 & 35 & 6,4 \\
\hline Erwerbstätige (Inland) & -39 & $-0,7$ & 118 & 2,1 \\
\hline Geförderte Erwerbstätige 3 ) & 17 & 7,6 & -15 & $-6,0$ \\
\hline Nicht geförderte Erwerbstätige & -56 & $-1,0$ & 133 & 2,5 \\
\hline \multicolumn{5}{|c|}{$\begin{array}{l}\text { 1) Arbeitsbeschaffungs- und Strukturanpassungsmaßnahmen; Beschäftigung schaffende Infrastrukturmaßnahmen; Arbeitnehmer } \\
\text { mit Eingliederungszuschüssen, Einstellungszuschüssen bei Vertretung, Einstellungszuschüssen bei Neugründung, Arbeitsentgelt- } \\
\text { zuschuss bei beruflicher Weiterbildung, Einstiegsgeld für abhängig Beschäftigte; 1-Euro-Job (Entgeltvariante. } \\
\text { 2) Gründungszuschuss, Ubberbrückungsgeld, Ich-AG, Einstiegsgeld (Variante: Selbstständigkeit). } \\
\text { 3) Geförderte SV-pflichtig Beschäftigte, 1-Euro-Jobs (Variante: Mehraufwandsentschädigung), geförderte Selbstständige. }\end{array}$} \\
\hline \multicolumn{5}{|c|}{$\begin{array}{l}\text { Quelle: Statistisches Bundesamt: Fachserie 1, Reihe 4.1.1; Arbeitskreis Erwerbstätigen- } \\
\text { rechnung des Bundes und der Länder; Bundesagentur für Arbeit; Berechnungen des IWH. }\end{array}$} \\
\hline
\end{tabular}

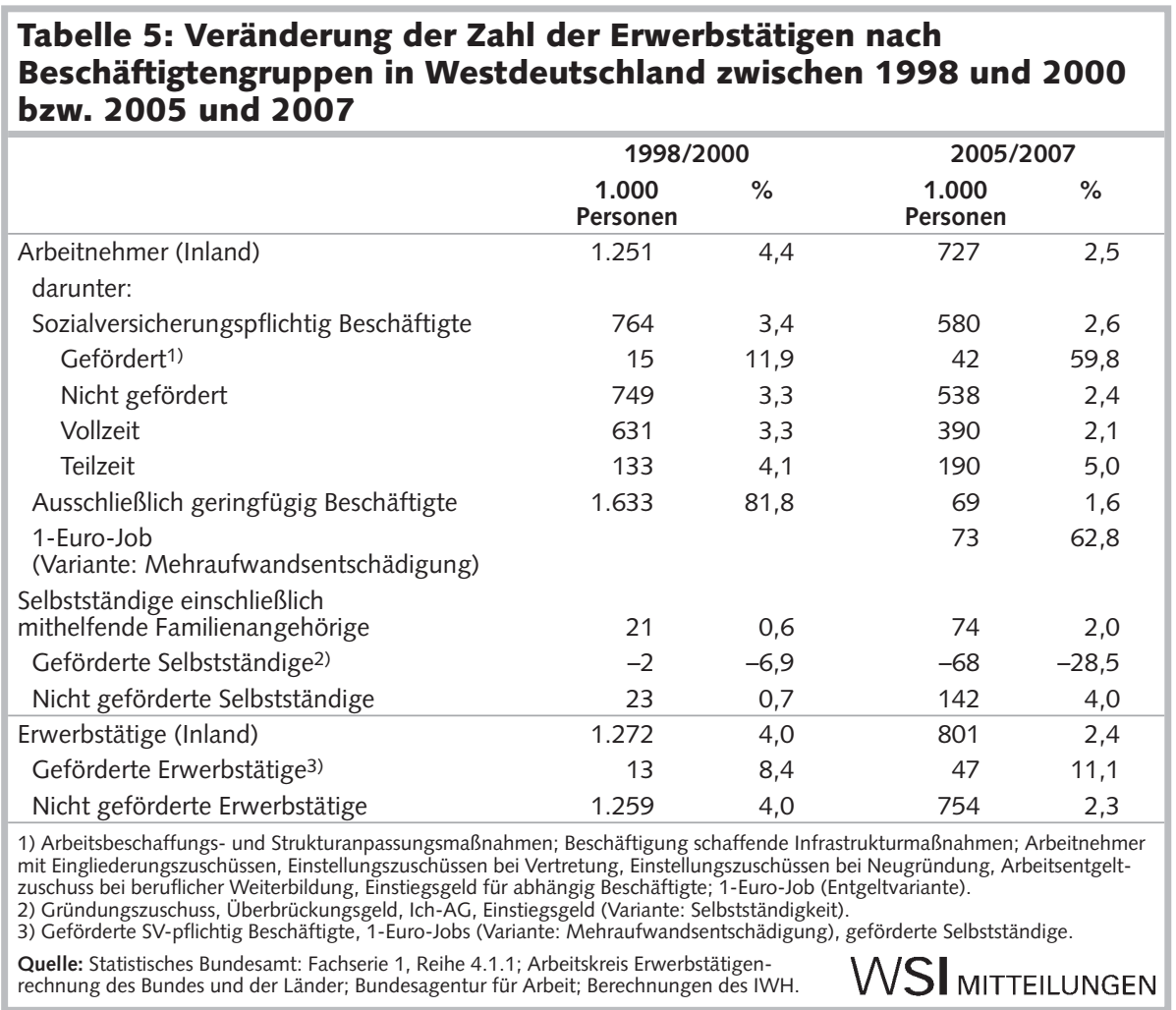

durch atypische Beschäftigungsverhältnisse, wie beispielsweise ausschließlich geringfügig entlohnte Beschäftigte und sozialversicherungspflichtig Teilzeitbeschäftigte, verdrängt wurden. Allerdings ist die Zunahme an Vollzeitarbeitsplätzen in Westdeutschland deutlich höher als in den neuen Bundesländern.

\section{Die Arbeitsplatzausstattung im Ost-West-Vergleich}

Bei einem Vergleich der Arbeitsplatzausstattung, die die Anzahl verfügbarer Ar- beitsplätze je 1000 Erwerbsfähige ausweist, zwischen Ost- und Westdeutschland muss berücksichtigt werden, dass zwischen 1991 und 2007 die Bevölkerung in Ostdeutschland - bedingt durch die demografische Entwicklung und die Wanderungsbewegungen - um 1,4 Mio. Personen bzw. 9,9 \% abgenommen hat. Die Zahl der Erwerbsfähigen sank in diesem Zeitraum in Ostdeutschland um etwa 780.000 Personen bzw. 8,1 \%. In Westdeutschland nahm sie nicht zuletzt durch die Wanderungsgewinne aus Ostdeutschland - um 350.000 Personen $(0,8 \%)$ zu. Bei der Untersuchung der Frage, in welchem Ausmaß Ost- bzw. Westdeutschland vom Aufschwung am Arbeitsmarkt partizipiert haben, muss der - an der Zahl der Erwerbsfähigen gemessene Rückgang des Arbeitsangebots berücksichtigt werden. Dies bedeutet, dass die zusätzlich geschaffenen Arbeitsplätze auf 1.000 Erwerbsfähige bezogen werden müssen. ${ }^{4}$ Ein Vergleich zwischen Ost- und Westdeutschland ergibt folgendes Bild (Tabelle 6): In Ostdeutschland nahm zwischen 2005 und 2007 die Zahl der Erwerbstätigen je 1.000 Erwerbsfähige um 35 Personen zu, in Westdeutschland waren es 21. Die Arbeitsplatzausstattung je 1000 Erwerbsfähige nahm zwischen den Jahren 2005 und 2007 in Ostdeutschland um 5,8 \% und in Westdeutschland um 2,9 \% zu. In der gleichen Größenordnung wuchs in Ostdeutschland auch das geleistete Arbeitsvolumen stärker an als in Westdeutschland. Dass sich die Arbeitsplatzausstattung in Ostdeutschland in diesem konjunkturellen Aufschwung stärker verbessert hat als in Westdeutschland, ist auf zwei Faktoren zurückzuführen: Zum einen hat die absolute Zahl der Erwerbstätigen zugenommen, und zwar um 118.000 Personen bzw. 2,1 \% (Tabelle 1), und zum anderen ging die Zahl der Personen, die potenziell Arbeitsplätze suchen, also die Personen im Alter von 15 bis 64 Jahre, um 323.000 Personen bzw. $-3,5 \%$ zurück. In Westdeutschland wuchs die Zahl der Erwerbstätigen mit 2,3\% zwar etwas stärker an; dagegen war der Rückgang der Erwerbsfähigen mit 0,5\% deutlich schwächer.

Betrachtet man die einzelnen Beschäftigungsgruppen, so wurde in beiden Großregionen der Arbeitsplatzbesatz vor allem der Großregionen müssten streng genommen auch der Zuwachs des gesamtwirtschaftlichen Stellenangebots einbezogen werden.
4 In die Berechnung der Arbeitsplatzausstattung bei- 
bei sozialversicherungspflichtig Beschäftigten und bei den Selbstständigen erhöht, wobei der Beitrag der staatlich geförderten Beschäftigungsverhältnisse gering war. Der Anteil, den die sozialversicherungspflichtig Teilzeitbeschäftigten sowie die ausschließlich geringfügig Beschäftigten am gesamten Zuwachs ausmachen, beträgt in beiden Großregionen etwa ein Drittel. Die Feststellung, dass sich die Qualität der Beschäftigungswirkung der gesamtwirtschaftlichen Expansion in Deutschland insgesamt im Vergleich zum vorangegangenen Aufschwung verbessert hat (Gartner/Klinger 2007, S. 618), gilt demnach für beide Großraumregionen.

Tabelle 7 macht veranschaulicht, wie sich die Arbeitsplatzausstattung in Ostdeutschland innerhalb der jüngsten Aufschwungphase weiterentwickelt hat. Kamen 2005 auf 1.000 Personen im erwerbsfähigen Alter 608 Erwerbstätige (Inland), so waren es zwei Jahr später bereits 643. Dies entsprach $84 \%$ bzw. $86 \%$ des westdeutschen Vergleichswerts. Damit ist die Arbeitsplatzausstattung in der Großregion Ostdeutschland trotz der aktuellen positiven Entwicklungen nach wie vor deutlich niedriger als in Westdeutschland. Zwischen den Beschäftigtengruppen gibt es jedoch erhebliche Unterschiede. Ein relativ hoher Anpassungsstand besteht mit $91 \%$ bei den sozialversicherungspflichtigen Vollzeitbeschäftigten, während bei den ausschließlich geringfügig entlohnten Beschäftigten nur $64 \%$ des westdeutschen Wertes erreicht werden. Bei der geförderten Erwerbstätigkeit hingegen wird in Ostdeutschland der westdeutsche Vergleichswert deutlich übertroffen.

Bei einem Vergleich der Arbeitsplatzausstattung muss allerdings beachtet werden, dass der Pendlersaldo zwischen Ostdeutschland und Westdeutschland etwa 400000 Personen zugunsten der neuen Bundesländer beträgt. Werden diese Pendlerströme in die Berechnung mit einbezogen, so kommen auf 1000 erwerbsfähige Ostdeutsche 688 Arbeitsplätze. Das entspricht $92 \%$ des westdeutschen Wertes.

Bei einem Vergleich der Arbeitsplatzausstattung muss auch die regional unterschiedlich hohe Jahresarbeitszeit eines Erwerbstätigen berücksichtigt werden. So war sie im Jahr 2007 in den neuen Bundesländern um knapp $6 \%$ höher als in Westdeutschland. Bezieht man dies in den Vergleich ein, so ergibt sich folgendes Bild: Das in Ostdeutschland verfügbare Arbeitsvolumen (in Stunden) je 1.000 Erwerbsfähige

\begin{tabular}{|c|c|c|c|c|}
\hline & \multicolumn{2}{|c|}{ Ostdeutschland } & \multicolumn{2}{|c|}{ Westdeutschland } \\
\hline & Personen & $\%$ & Personen & $\%$ \\
\hline $\begin{array}{l}\text { Arbeitnehmer (Inland) } \\
\text { darunter: }\end{array}$ & 31 & 5,7 & 19 & 3,0 \\
\hline Sozialversicherungspflichtig Beschäftigte & 28 & 6,4 & 15 & 3,1 \\
\hline Gefördert¹) & -1 & $-9,1$ & 1 & 60,5 \\
\hline Nicht gefördert & 28 & 6,8 & 14 & 2,9 \\
\hline Vollzeit & 20 & 5,4 & 11 & 2,6 \\
\hline Teilzeit & 8 & 11,8 & 5 & 5,5 \\
\hline Ausschließlich geringfügig Beschäftigte & 4 & 6,2 & 2 & 2,1 \\
\hline $\begin{array}{l}\text { 1-Euro-Job } \\
\text { (Variante: Mehraufwandsentschädigung) }\end{array}$ & 2 & 21,8 & 2 & 63,6 \\
\hline $\begin{array}{l}\text { Selbstständige einschließlich } \\
\text { mithelfende Familienangehörige }\end{array}$ & 4 & 6,4 & 2 & 2,5 \\
\hline Geförderte Selbstständige2) & -2 & $-18,4$ & -1 & $-28,2$ \\
\hline Nicht geförderte Selbstständige & 6 & 10,3 & 3 & 4,5 \\
\hline Erwerbstätige (Inland) & 35 & 5,8 & 21 & 2,9 \\
\hline Geförderte Erwerbstätige ${ }^{3)}$ & -1 & $-2,6$ & 1 & 11,6 \\
\hline \multirow[t]{2}{*}{ Nicht geförderte Erwerbstätige } & 36 & 6,2 & 20 & 2,8 \\
\hline & Stunden & $\%$ & Stunden & $\%$ \\
\hline Arbeitsvolumen (Inland) & 49 & 5,4 & 29 & 2,8 \\
\hline Arbeitsvolumen (Inländer) & 53 & 5,4 & 29 & 2,9 \\
\hline \multicolumn{5}{|c|}{$\begin{array}{l}\text { 1) Arbeitsbeschaffungs- und Strukturanpassungsmaßnahmen; Beschäftigung schaffende Infrastrukturmaßßßnahmen; Arbeitnehmer } \\
\text { mit Eingliederungsuzuschüssen, Einstellungszuschüssen bei Vertretung, Einstellungszuschüssen bei Neugründung, Arbeitsentgelt- } \\
\text { zuschuss bei beruflicher Weiterbildung, Einstiegsgeld für abhängig Beschäftigte; } 1 \text {-Euro-Job (Entgeltvariante). } \\
\text { 2) Gründungszuschuss, Überbrückungsgeld, Ich-AG, Einstiegsgeld (Variante: Selbständigkeit). } \\
\text { 3) Geförderte SV-pflichtig Beschäftige, 1-Euro-Jobs (Variante Mehraufwandsentschädigung), geförderte Selbstständige. }\end{array}$} \\
\hline $\begin{array}{l}\text { Quelle: Arbeitskreis Erwerbstätigenrechnung des Bund } \\
\text { Bundesagentur für Arbeit; Berechnungen des IWH. }\end{array}$ & ler Länder; & & SI MITT & LUNGE \\
\hline
\end{tabular}

Tabelle 7: Arbeitsplatzausstattung in Ost- und Westdeutschland 2005 und 2007 - Personen je 1.000 Erwerbsfähige -

\begin{tabular}{|c|c|c|c|c|}
\hline & \multicolumn{2}{|c|}{ Ostdeutschland } & \multicolumn{2}{|c|}{ Westdeutschland $=100$} \\
\hline & 2005 & 2007 & 2005 & 2007 \\
\hline $\begin{array}{l}\text { Arbeitnehmer (Inland) } \\
\text { darunter: }\end{array}$ & 541 & 572 & 84 & 86 \\
\hline Sozialversicherungspflichtig Beschäftigte & 429 & 457 & 88 & 91 \\
\hline Gefördert11) & 9 & 8 & 599 & 339 \\
\hline Nicht gefördert & 420 & 449 & 87 & 90 \\
\hline Vollzeit & 362 & 381 & 89 & 92 \\
\hline Teilzeit & 68 & 76 & 83 & 87 \\
\hline Ausschließlich geringfügig Beschäftigte & 57 & 61 & 62 & 64 \\
\hline $\begin{array}{l}\text { 1-Euro-Job } \\
\text { (Variante: Mehraufwandsentschädigung) }\end{array}$ & 8 & 10 & 326 & 243 \\
\hline $\begin{array}{l}\text { Selbstständige einschließlich mithelfende } \\
\text { Familienangehörige }\end{array}$ & 67 & 71 & 82 & 85 \\
\hline Geförderte Selbstständige2) & 9 & 7 & 174 & 197 \\
\hline Nicht geförderte Selbstständige & 58 & 64 & 76 & 80 \\
\hline Erwerbstätige (Inland) & 608 & 643 & 84 & 86 \\
\hline Geförderte Erwerbstätige 3 ) & 27 & 26 & 286 & 249 \\
\hline Nicht geförderte Erwerbstätige & 581 & 617 & 81 & 84 \\
\hline \multirow[t]{2}{*}{ Erwerbstätige (Inländer) } & 650 & 688 & 91 & 93 \\
\hline & \multicolumn{4}{|c|}{ Stunden } \\
\hline Arbeitsvolumen (Inland) & 916 & 965 & 89 & 91 \\
\hline Arbeitsvolumen (Inländer) & 976 & 1.029 & 96 & 98 \\
\hline \multicolumn{5}{|c|}{$\begin{array}{l}\text { 1) Arbeitsbeschaffungs- und Strukturanpassungsmaßnahmen; Beschäftigung schaffende Infrastrukturmaßnnahmen; Arbeitnehmer } \\
\text { mit Eingliederunguzzuschüssen, Einstellungszuschüssen bei Vertretung, Einstellungszuschüssen bei Neugründung, Arbeitsentgelt- } \\
\text { zuschuss bei be-ruflicher Weiterbildung, Einstiegsgeld für abhängig Beschäftigte; } 1 \text {-Euro-Job (Entgeltvariante). } \\
\text { 2) Gründungszuschuss, Uberbrückungsgeld, Ich-AG, Einstiegsgeld (Variante: Selbständigkeit). } \\
\text { 3) Geförderte SV-pflichtig Beschäftige, 1-Euro-Jobs (Variante Mehraufwandsentschädigung), geörderte Selbstständige. }\end{array}$} \\
\hline \multicolumn{3}{|c|}{$\begin{array}{l}\text { Quelle: Arbeitskreis Erwerbstätigenrechnung des Bundes und der Länder; } \\
\text { Bundesagentur für Arbeit; Berechnungen des IWH. }\end{array}$} & VSIM & LUNGEN \\
\hline
\end{tabular}

beträgt $91 \%$ des westdeutschen Vergleichswertes. Bei Einrechnung der - in Arbeitsstunden - ausgedrückten Pendlerströme ergibt sich ein Anpassungsstand von $98 \%$. Dieser hohe Anpassungsstand kommt nur dadurch zustande, dass erheblich mehr Ostdeutsche nach Westdeutschland pendeln als umgekehrt und die Jahresarbeitszeit in Ostdeutschland höher ist als in den alten Bundesländern. 


\section{Arbeitsplatzlücke in Ostdeutschland doppelt so hoch}

In Ostdeutschland besteht nach wie vor eine große Diskrepanz zwischen dem Angebot und der Nachfrage nach Arbeitsplätzen. Einem Angebot an nichtsubventionierten Arbeitsplätzen im ersten Arbeitsmarkt in Höhe von 5,9 Mio. im Jahr 2007 steht ein Defizit von mehr als 1,2 Mio. Arbeitsplätzen gegenüber (Tabelle 8 ). Diese rechnerische Arbeitsplatzlücke ergibt sich summarisch aus der Zahl der Unterbeschäftigten und der Zahl der Personen, die zur sogenannten „Stillen Reserve im engeren Sinne" zählen, ${ }^{5}$ abzüglich des gesamtwirtschaftlichen Stellenangebots. Zur „Stillen Reserve im engeren Sinne" gehören Personen, die beschäftigungslos sind und Arbeit suchen, ohne bei den Arbeitsagenturen als arbeitslos registriert zu sein, sowie Personen, die bei ungünstiger Lage auf dem Arbeitsmarkt die Arbeitsuche entmutigt aufgegeben haben, aber bei guter Arbeitsmarktlage einen Arbeitsplatz suchen (BA 2006, S. 73). Die Zahl der Personen, die zur "Stillen Reserve im engeren Sinne" zählen, beträgt in Ostdeutschland etwa 122.000 Personen. ${ }^{6}$ Bei der Berechnung der rechnerischen Arbeitsplatzlücke müssen die freien Stellen abgezogen werden, da diese durch Arbeitsuchende besetzt werden könnten, die hinsichtlich ihres Berufes, ihrer Qualifikation und ihrer regionalen Verfügbarkeit geeignet sind.

Bezieht man diese Arbeitsplatzlücke auf die Zahl der Erwerbsfähigen, dann ergibt sich folgendes Bild: In Ostdeutschland fehlten im Jahr 2007 je 1.000 Erwerbsfähige 139 Arbeitsplätze am ersten Arbeitsmarkt, in den alten Bundesländern waren es 62. Im Jahr 2005 waren es noch 177 in Ostdeutschland bzw. 80 in Westdeutschland. Die Arbeitsplatzlücke ist im gegenwärtigen konjunkturellen Verlauf in beiden Großraumregionen deutlich zurückgegangen. Sie ist aber in Ostdeutschland immer noch mehr als doppelt so hoch wie in Westdeutschland.

Angesichts einer Arbeitsplatzausstattung der erwerbstätigen Inländer, die $93 \%$ des westdeutschen Wertes erreicht, überrascht die doppelt so hohe Arbeitsplatzlücke in Ostdeutschland. Dies hat zwei Ursachen: ${ }^{7}$ Zum ersten stehen den Ostdeutschen 688, den Westdeutschen 736 Arbeitsplätze je 1.000 Erwerbsfähigen zur Verfügung (Tabelle 9). Damit verfügen die Ostdeutschen um 48 Arbeitsplätze je 1.000 Erwerbsfähige weniger als die Westdeutschen. Bei 8,9 Mio. Erwerbsfähigen entspricht das etwa 427.000 Personen. ${ }^{8}$ Dies ist die Zahl an Arbeitsplätzen, die den Ostdeutschen bei einer gleich hohen Arbeits-

\begin{tabular}{|c|c|c|c|c|}
\hline & \multicolumn{2}{|c|}{ Ostdeutschland } & \multicolumn{2}{|c|}{ Westdeutschland } \\
\hline & \multicolumn{4}{|c|}{1.000 Personen } \\
\hline & 2005 & 2007 & 2005 & 2007 \\
\hline Unterbeschäftigte & 1.572 & 1.297 & 4.138 & 3.378 \\
\hline + Stille Reserve im engeren Sinne ${ }^{1)}$ & 190 & 122 & 529 & 475 \\
\hline - Stellenangebot & 132 & 182 & 981 & 1.040 \\
\hline $\begin{array}{l}=\text { Arbeitslücke insgesamt } \\
\text { (= Unterbeschäftigung im weiteren Sinne) }\end{array}$ & 1.630 & 1.237 & 3.686 & 2.813 \\
\hline Erwerbsfähige2) & 9.223 & 8.900 & 45.840 & 45.620 \\
\hline Anteil der Arbeitslücke an den Erwerbsfähigen in \% & 17,7 & 13,9 & 8,0 & 6,2 \\
\hline \multicolumn{5}{|c|}{$\begin{array}{l}\text { 1) Dazu zählen Personen, die beschäftigungslos sind und Arbeit suchen, ohne bei den Arbeitsagenturen als arbeitslos registriert } \\
\text { zu sein sowie Personen, die bei ungünstiger Lage auf dem Arbeitsmarkt die Arbeitssuche entmutigt aufgegeben haben, aber bei } \\
\text { guter Arbeitsmarktlage einen Arbeitsplatz suchen. } \\
\text { 2) Personen im Alter von } 15 \text { bis } 64 \text { Jahren. }\end{array}$} \\
\hline $\begin{array}{l}\text { Quelle: Statistisches Bundesamt: Fachserie 1, Reihe 4.1.1; Bund } \\
\text { Bach et.al. 2008; Kettner/Spitznagel 2008; Berechnungen und S }\end{array}$ & ntur für & & & 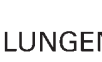 \\
\hline
\end{tabular}

\begin{tabular}{|c|c|c|c|}
\hline & Ostdeutschland & Westdeutschland & Differenz \\
\hline Erwerbstätige (Inländer) & 688 & 736 & -48 \\
\hline Unterbeschäftigte im weiteren Sinne & 139 & 62 & 77 \\
\hline Insgesamt & 827 & 798 & 29 \\
\hline
\end{tabular}

platzausstattung wie in Westdeutschland zusätzlich zur Verfügung stünden. Sie ist Ausdruck des wirtschaftlichen Gefälles zwischen beiden Großregionen.

Die zweite Ursache besteht darin, dass die Erwerbsbeteiligung der Ostdeutschen nach wie vor höher ist als die der Westdeutschen. Je 1.000 erwerbsfähige Ostdeutsche gibt es 827 Personen, die eine Erwerbstätigkeit im ersten Arbeitsmarkt ausüben bzw. suchen (688 Personen je 1.000 in Ostdeutschland haben einen Arbeitsplatz, 139 je 1.000 suchen einen). Die Erwerbsbeteiligung je 1.000 Erwerbsfähige ist in Ostdeutschland damit um 29 Personen höher als in Westdeutschland. Bezogen auf alle ostdeutschen Erwerbsfähigen sind dies 258.000 Personen. Mit anderen Worten: Bei einem gleich hohen Angebot an wettbewerbsfähigen Arbeitsplätzen und einer Erwerbsbeteiligung in Ostdeutschland, die so hoch ist wie in Westdeutschland, wäre die - auf die Erwerbsfähigen bezogene Arbeitsplatzlücke so groß wie in den alten Bundesländern.

\section{Fazit}

Der Aufschwung der Jahre 2005-2007 hat das Beschäftigungsniveau in Ostdeutschland erhöht, und die Unterbeschäftigung ist deutlich zurückgegangen. Die Arbeitsplatzausstattung hat sich in Ostdeutschland weiter dem westdeutschen Vergleichswert angenähert. Dies ist zum einen dem Beschäftigungsaufbau geschuldet. Zum anderen führt der Rückgang der Zahl der Erwerbsfähigen statistisch zu einer Zunahme der Arbeitsplatzausstattung. Die mehr als doppelt so hohe Arbeitsplatzlücke ist sowohl auf ein fehlendes Angebot an wettbewerbsfähigen Arbeitsplätzen als auch auf eine höhere Erwerbsbeteiligung zurückzuführen.

5 Die Arbeitsplatzlücke würde noch höher ausfallen, wenn die Personen mit einbezogen würden, die unfreiwillig, d.h. in Ermangelung des Angebots einer Vollzeitstelle, einer Teilzeitarbeit nachgehen (Brautzsch et al. 2007).

6 Geschätzt auf der Grundlage der Angaben bei Bach et.al. 2008.

7 Eine ausführliche Diskussion der Ursachen ist zu finden bei Brautzsch 2004; Brautzsch et al. 2007.

8 Dies ergibt sich folgendermaßen: $8.900 .000 \mathrm{Er}$ werbsfähige * 48/1.000 Erwerbsfähige $=427.200$ 
Arbeitskreis Erwerbstätigenrechnung des Bundes und der Länder (2008): Erwerbstätige in den Ländern der Bundesrepublik Deutschland 1991 bis 2007

Arbeitskreis Volkswirtschaftliche Gesamtrechnungen der Länder (2008): Bruttoinlandsprodukt in den Ländern der Bundesrepublik Deutschland 1991 bis 2007

Arbeitskreis Konjunktur Ostdeutschland (2008): Ostdeutsche Wirtschaft: Kein aufholendes Produktionswachstum 2008 und 2009, in: Wirtschaft im Wandel 6, S. 205-236

Bach, H.-U./Gartner, H./Klinger, S./Rothe, T./ Spitznagel, E. (2007): Arbeitsmarkt 2007/2008: Ein robuster Aufschwung mit freundlichem Gesicht, in: IAB-Kurzbericht 15, S. 1-8

Bach, H.-U./Gartner, H./Klinger, S./Rothe, T./ Spitznagel, E. (2008): Arbeitsmarkt 2008: Der Aufschwung lässt nach, IAB-Kurzbericht 3, S. 1-12

Brautzsch, H.-U. (2004): Arbeitsplatzlücke im Osten fast doppelt so hoch wie im Westen, Wirtschaft im Wandel 11, S. 295-300

Brautzsch, H.-U./Fuchs, J./Lang, C. (2007): Wie hoch ist die Unterbeschäftigung in Ost- und Westdeutschland? Arbeitsplatzausstattung und Arbeitsplatzlücke in Ost- und Westdeutschland, in: Wirtschaftspolitische Blätter 1, S. 375-393
Bundesagentur für Arbeit (BA) (2006): Arbeitsmarkt 2006

Gartner, H./Klinger, S. (2007): Aufschwung am Arbeitsmarkt - Trendwende oder Streufeuer?, in: Wirtschaftsdienst 9, S. 613-619

Horn, G./Logeay, C./Zwiener, R. (2008): Wer profitierte vom Aufschwung?, in: IMK Report 27, S. 1-14

Institut für Arbeitsmarkt- und Berufsforschung (IAB) (2008): Daten zur kurzfristigen Entwicklung von Wirtschaft und Arbeitsmarkt - Juni 2008 Kettner, A./Spitznagel, E. (2008): Stellenangebot geht zurück, bleibt aber auf hohem Niveau, in: IAB-Kurzbericht 7, S. 1-8

Kubis, A./Schneider, L. (2008): Wanderungsverhalten der Ostdeutschen, in: Wirtschaft im Wandel 4, S. 128-131

Ludwig, U. (2008): Konjunktur in Deutschland seit der Vereinigung, in: Konjunkturperspektiven, S. 13-23 ((Bitte Heft-Nr. angeben))

Magvas, E./Spitznagel, E. (2002): Deutliche Bremsspuren auch im gesamtwirtschaftlichen Stellenangebot, in: IAB-Kurzberichte 14, S. 1-9 Projektgruppe Gemeinschaftsdiagnose (2008): Folgen der US-Immobilienkrise belasten Konjunktur, Kiel

Sachverständigenrat zur Begutachtung der gesamtwirtschaftlichen Entwicklung (SVR) (2007): Jahresgutachten 2007/2008, Das Erreichte nicht verspielen, Kasten 14, S. 325-326, Wiesbaden 


\section{ZUSAMMENFASSUNGEN}

\section{BRAUTZSCH, HANS-ULRICH}

Zur Entwicklung des ostdeutschen Arbeitsmarktes im Aufschwung

in: WSI-Mitteilungen 9/2008, Seiten

Der kräftige konjunkturelle Aufschwung der Jahre 2005-2007 hat auch den ostdeutschen Arbeitsmarkt erreicht. So nahm in Ostdeutschland die Zahl der Erwerbstätigen das zweite Jahr in Folge zu und die Unterbeschäftigung ging deutlich zurück. Der Beitrag untersucht einige Aspekte dieser Entwicklung vertiefend. Als Vergleichsmaßstab dient zum einen der vorangegangene Aufschwung (1998-2000), zum anderen die Arbeitsmarktentwicklung in Westdeutschland. In einem ersten Schritt wird anhand der Indikatoren Erwerbstätigenzahl, Arbeitsvolumen und Anzahl der Unterbeschäftigten analysiert, in welchem Umfang sich die Lage auf dem ostdeutschen Arbeitsmarkt verbessert hat. Anschließend wird der Beschäftigungsstand bei einzelnen Beschäftigtengruppen, wie beispielsweise bei den sozialversicherungspflichtigen Erwerbstätigen oder den Selbstständigen, untersucht. Danach wird aufgezeigt, wie sich im gegenwärtigen konjunkturellen Aufschwung die Arbeitsplatzausstattung - also die Anzahl verfügbarer Arbeitsplätze - entwickelt hat. Die Analyse mündet in der Feststellung, dass trotz der Verbesserung der Arbeitsplatzausstattung die Arbeitsplatzlücke nach wie vor hoch ist. 